\title{
Hybrid Regression Analysis based Technique for Removal of Impulse Noise from Gray-Scale Images
}

\author{
Deepshri Wagh \\ Department of IT \\ Medi-Caps Institute of Technology and \\ Management, Indore (M.P)
}

\author{
C.S. Satsangi, $\mathrm{PhD}$ \\ Department of IT \\ Medi-Caps Institute of Technology and \\ Management, Indore (M.P)
}

\begin{abstract}
Noise is major limiting factor in digital images, due to noise quality of image is degrade. For improving quality of image filtering techniques are used. There are various filtering technique are reported in last few years. In this presented work the impulse noise and their effect in image is measured. In addition of that the recent contributions on the image filters for impulse noise detection and correction is also investigated. The Filtering algorithms considered are: Fuzzy Logic Based Adaptive Noise Filter [1], Cloud Model Filter [2]. Several runs on many images were made using these algorithms. Whereas the noise detection process of the CM filter was good, and the correction process of Fuzzy Logic Based Adaptive Noise Filter was better. By combining the concepts of both these algorithms and also using some additional concept like regression analysis, L ZERO smoothing, an improved impulse noise filtering algorithm is developed. Proposed algorithm enhances the image quality. This algorithm is iterative, so as much iteration as the user may desire can be made considering image quality. The performance of the proposed algorithm is measure in term of visual quality and PSNR.
\end{abstract}

\section{General Terms}

Error Filtering, Image Processing, Image Enhancement, Performance Improvement.

\section{Keywords}

Regression analysis, L ZERO smoothing, PSNR.

\section{INTRODUCTION}

Noise is random variation of brightness and color information in image. Whenever noise occurs, it adds some spurious and extraneous information in the image. There are several reasons for occurrence of noise in images such as: digitizing, scanning, transmission, storage, etc... There are several types of noises that occur in images such as Gaussian, Impulse, Poisson, and Multiplicative. Among these types of noises, Impulse noise is generally found in all images.

Impulse noise occurs because of channel decoder damages, dyeing down of signal from communication link, video sensor noise etc... Impulse noise is also called Salt and Pepper noise. Because of impulse noise, white and black spots appear in the digital gray scale image. Salt \& Pepper noise significantly degrades the image quality.

A gray scale image represented by a two-dimensional array where a location $(i, j)$ is a position in image. Often the gray scale image is stored as an 8-bit integer that gives 256 possible different shades of gray going from black to white. Pixels can have value in [0-255] integer interval, but some pixels in an image do not have a correct value because of noise. On another hand (i, j) may include impulse noises such as pepper (255) and salt (0) [3]

\section{BACKGROUND AND MOTIVATION}

Over the last years great amount of work has been done to detect and remove impulse noise from gray scale images.

Jasdeep et al [1] proposed a method and the name of the method is Fuzzy Logic Based Adaptive Noise Filter, this method consist of three steps, in the first step, center pixel of the window is tested whether pixel is affected by impulse noise or not, detected pixel affected by impulse noise then it lies outside from the trimming range by using fuzzy reasoning. In the second step, replace the noisy pixels by using median filters. In third step, create a histogram of the image and again remove the noise by using soft thresh holding. The Result of the algorithm calculated in term of PSNR value.

The steps of the algorithm are described as bellow.

\section{Algorithm 1}

1. Read the gray scale image.

2. Add the "salt pepper" noise to the image.

3. $3 * 3$ median filters are used to remove the noise from the gray scale noisy image.

4. $3 * 2$ median filters by using fuzzy logic are used to remove the noise from the edges of the image.

5 . $4 * 4$ median filter is used by using fuzzy logic to improve the quality of the gray scale image. Also create histogram of the image.

6. Again create histogram of the previous image by using thresh holding concept to remove the remaining noise from the gray scale image.

Fuzzy Logic Based Adaptive Noise Filter provides good results in low percentage of noise in term of PSNR value, but this filter is not suitable for high level of noise.

Zhe Zhou [2] proposed a method and the name of method is Cloud Model filter, this method presents a novel adaptive detail-preserving filter for removing impulse noise from gray scale images. In this algorithm an uncertainty-based detector identifies which pixels are affected by impulse noise. Then, a Weighted Fuzzy Mean Filter is used for correction of noisy pixel from gray scale image. The result of the algorithm is calculated in terms of PSNR value. 
The Steps of the detection (noisy pixel) algorithm are described in Algorithm 2

\section{Algorithm 2:}

Initialize $N=1$ and threshold $\delta(\delta$ is positive integer), denote $n$ as the number of uncorrupted pixel in $W_{i, j}^{2 N+1}$, and initialize $n=0$.

1. Calculate $E x$ of all pixel in $W_{i, j}^{2 N+1}$,i.e.

$$
E x=\frac{1}{n} \sum_{x_{i+s, i+t} \in W_{i, j}^{2 N+1}} x_{i+s, j+t .}
$$

2. Calculate $E n$, i.e.

$$
E n=\sqrt{\frac{\pi}{2}} * \frac{1}{n} \sum_{x_{i+s, i+t \in W_{i, j}^{2 N+1}}}\left|x_{i+s, j+t}-E X\right| .
$$

3. Calculate $W_{\max }^{2 N+1}$ and $W_{\min }^{2 N+1}$, respectively i.e.

$$
\begin{aligned}
& W_{\max }^{2 N+1}=\operatorname{Min}\left(S_{\max }, E x+3 E n\right) \\
& W_{\min }^{2 N+1}=\operatorname{Max}\left(S_{\min }, E x-3 E n\right)
\end{aligned}
$$

Where Max and Min are the extreme operations to recover the smallest and the largest of the two values.

5. If $W_{\text {min }}^{2 N+1}<x_{i, j}<W_{\max }^{2 N+1}$ then $x_{i, j}$ is an uncorrupted pixel; otherwise, go to step 6 .

6. Identify if the other pixels in $W_{i, j}^{2 N+1}$ are the noise pixels or not. For pixel $x_{i+s, j+t}$, if $W_{\text {min }}^{2 N+1}<x_{i+s, j+t}<W_{\text {max }}^{2 N+1}$, then $x_{i+s, j+t}$ will remain and $n=n+1$.

7. If $W_{\min }^{2 N+1} \geq x_{i, j}$ or $x_{i, j} \geq W_{\max }^{2 N+1}$, with $n<\delta$, set $N=N+1$ and go to step 2; otherwise, $x_{i, j}$ noise candidat

The Steps of the correction (noisy pixel) algorithm are described in algorithm 3.

\section{Algorithm 3}

1. Calculate Ex of each uncorrupted pixel in $N_{i, j}^{2 N+1}$ ,i.e.

$$
E x=\frac{1}{n} \sum_{x_{i+s, i+t} \in N_{i, j}^{2 N+1}} x_{i+s, j+t .}
$$

2. Calculate En, i.e.,

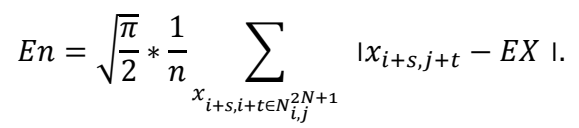

3. Calculate weights for $x_{i+s, j+t}$, i.e. $w_{i+s, j+t}=\exp \left(-\left(x_{i+s, j+t}-E x\right)^{2} / 2 E n^{2}\right)$.

4. Calculate the weighted mean, i.e.

$$
y_{i, j}=\sum_{x_{i+s, i+t} \in N_{i, j}^{2 N+1}} w_{i+s, j+t .} x_{i+s, j+t .}
$$

\section{LITERATURE REVIEW}

This has been observed by investigation of different techniques there are some methods available to reduce impulse noise from gray scale images. Furthermore by review of their results the determined pros and cons are listed below.

In this paper [1] author suggested Fuzzy Logic Based Adaptive Noise Filter for removing Impulse Noise from gray scale images. This filter uses median filter with different window size for removing impulse noise. This filter provides good results in terms of PSNR value with low percentage of noise but algorithm is computationally expensive and does not provide good result in higher percentage of noise.

CM Filter [2] uses an uncertainty-based detector identifies which pixels are affected by impulse noise. Then, a Weighted Fuzzy Mean Filter is used for correction of noisy pixel from gray scale image. This filter is easy to implement and in small size of image filter provide result in shorter time, but image size is large this filter consume more time and filter does not preserve edges sharpness.

The detection process of Standard devation based algorithm [4] based on finding optimum direction in the filtering window. The tested pixel is deemed original if it is similar to the pixels in the optimum direction. This algorithm removes noise preserving edges sharpness but this algorithm consumes most of time for detection between corrupted and uncorrupted pixels.

Modified Decision Based Unsymmetrical Trimmed Median Filter [5] uses trimmed value for replace corrupted pixels. This algorithm is easy to implement and remove noise from gray scale as well as color images. But this method not preserves edges sharpness in higher percentage of noise.

Improved fuzzy based decision algorithm [6] utilized modified version of the detection phase of FBDA to get better image quality. This method provides good quality in higher percentage of noise. But method does not provide good quality in higher percentage of noise.

Noise Adaptive Fuzzy Switching Median Filter [7] uses twostage adaptive fuzzy switching filter for noise removal. This method is able to suppress high-density of salt-and-pepper noise, at the same time preserving fineness of image details, edges and textures well. But method require more time in higher percentage of noise.

Patch-based nonlocal operator with noise detection [9] uses Adaptive median filter for detection than learn patch-based sparse representation. Patch-based sparse is learnt from image. In lasts a weighted regularization method is proposed penalize the noise candidates. This method is good for preserving edges sharpness and provides higher structure similarity with noise-free image. When percentage of noise is higher non local similarity is not higher.

Modified Non-linear Filter algorithm [10] first detect corrupted and uncorrupted pixel than corrupted pixels are processed to replace with noise free pixel value, uncorrupted pixels are left unchanged. This algorithm is applicable on gray as well as color images. But in higher percentage of algorithm does not preserve edges sharpness.

Quantized Adaptive Switching Median Filter (QASMF) [11], is derived from simple Adaptive Median Filter QASMF is constructed from two main processing blocks. In the first block noisy image is filtered using adaptive switching median filter and utilized quantized window size. The second block 
uses Simple adaptive median filter for filter noise. QASMF has better local content preservation ability. When noise density is high algorithm require more time.

\section{PROPOSED ALGORITHM}

The distribution of noise over an image, in the case of impulse noise, is never similar. In addition to that, the most of the filtering algorithms with high level of noise, compromised with their edges during recovery of the image, leading to significantly degraded images.

In order to reduce the above given deficiencies, a new hybrid algorithm is designed to implement. Proposed algorithm uses the good qualities of base algorithms. During impulse noise the image pixels are corrupted therefore to distinguish between corrupted and uncorrupted pixels the proposed algorithm uses regression analysis. After differentiation of corrupted pixel, these pixels are processed by calculating average value of itself and two neighboring pixels which are uncorrupted. Corrupted pixels are not properly removed in single processing therefore in iterative manner the corrupted pixel is removed from image. This processing can damage the image edges therefore in order to preserve the image edges L0 smoothing is employed in each iteration. Finally for removing the remaining noise effect a median filter is applied over the image. The implementation of the proposed algorithm is performed using MATLAB simulation environment.

\section{The steps of the proposed algorithm is given in Algorithm} 4.

\section{Algorithm 4}

Input : noisy image I, number of iterations $n_{i}$

Output: filtered image $I^{\prime}$

Process:

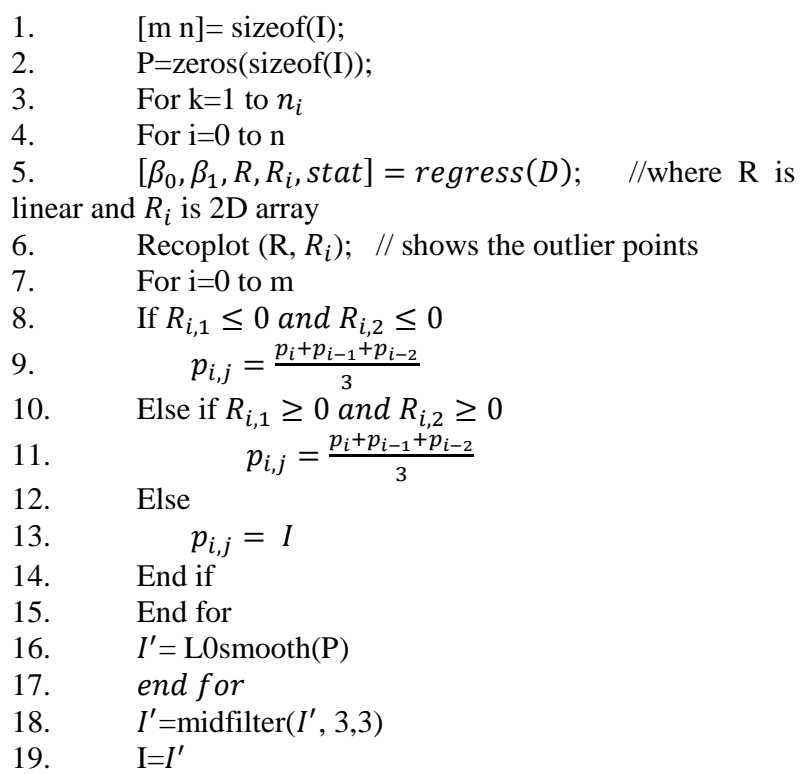

\subsection{Regression Analysis}

Regression analysis is a statistical process for estimating the relationships among variables, suppose there exists some relationship between two variables $\mathrm{x}$ and $\mathrm{y}$, the dots of scatter diagram will be more or less cluster about a curve. This curve is called the curve of regression.

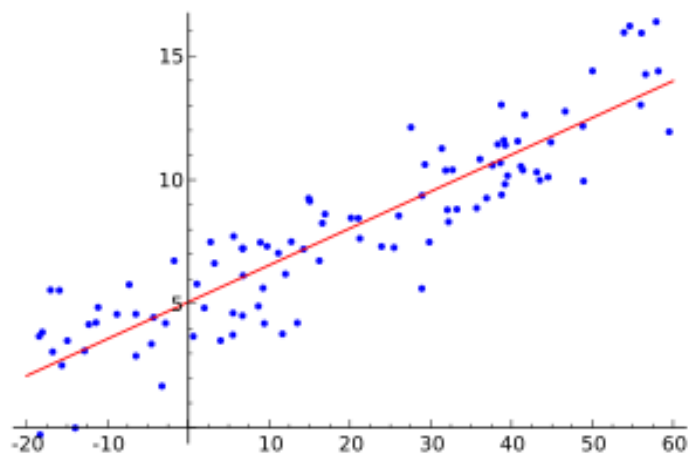

Fig (1) Illustration of linear regression

\subsection{Zero Smoothing:}

L Zero Smoothing is used for sharpening major edges by increasing the steepness of transition while eliminating a manageable degree of low-amplitude structures. The main contribution of L Zero Smoothing is to confine the discrete number of intensity changes among neighboring pixels [8]

\section{RESULT ANALYSIS}

In order to demonstrate performance of the proposed algorithm, some experiments were performed at different noise levels ranging from $10 \%$ to $70 \%$. The Performance rate was measured in terms of visual quality and peak-signal-tonoise-ratio (PSNR).

\subsection{Visual Quality}

The experimentation of presented techniques is performed on different noise levels of gray- scale images. Fuzzy Logic Based Adaptive Noise Filter is able to remove impulse noise up to $40 \%$ of noise composition, when composition or density of noise is higher than $40 \%$ than some noise candidates are remain see in fig(5). On the other hand CM (cloud model) Filter is good for removal of higher percentage of noise but when image size is large then requires time for process the noisy image is also larger. Additionally that can affect the edges of input image see in fig (6). The proposed technique provides good result even in higher percentage of noise up to $70 \%$ is introduced in input image. This technique also able to preserving edges and sharpness see in fig (4).

End for 


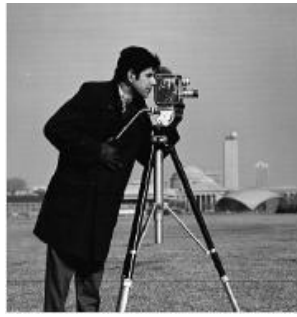

$\operatorname{Fig}(2)$

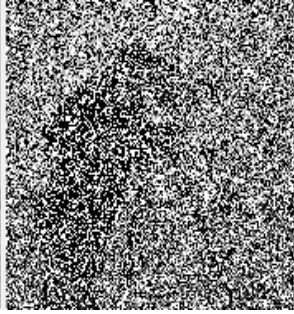

$\operatorname{Fig}(3)$

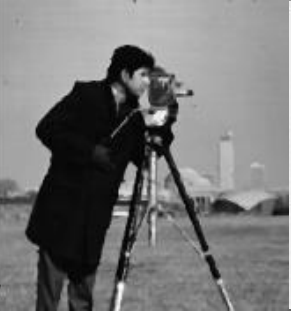

$\operatorname{Fig}(4)$

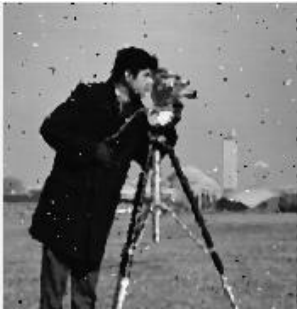

$\operatorname{Fig}(5)$

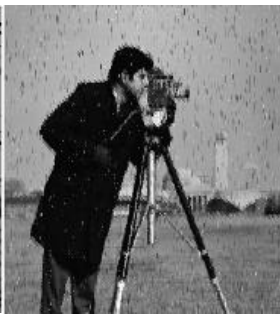

$\operatorname{Fig}(6)$

Fig: Original gray-scale Cameraman image. Fig (2) Cameraman image corrupted by 70\% impulse noise. Fig (3) Represents output of the proposed algorithm. Fig (4) Represents output of Fuzzy Logic Based Adaptive Median Filter. Fig (5) Represent output of Cloud Model

\subsection{PSNR (Peak signal to noise ratio)}

The PSNR measures the peak signal-to-noise ratio between two images. This ratio is often used as a quality measurement between the original and a compressed image. Higher the PSNR means better the quality of the compressed or reconstructed image. The PSNR value can be calculated as:

$$
P S N R=10 \log _{10} \frac{255^{2}}{\frac{1}{M N} \sum_{i, j}\left(y_{i, j}-x_{i, j}\right)^{2}}
$$

Where $y_{i, j}$ and $x_{i, j}$ denotes pixel value of original image and restored image.

The proposed algorithm is iterative, due to this reason PSNR is calculated in different iteration. In lower percentage of noise $(10 \%$ to $30 \%)$ one iteration is enough to achieve good quality. In medium percentage of noise ( $40 \%$ to $50 \%$ ) two or three iteration is required and higher percentage of noise $(60 \%$ to $70 \%$ ) uses four or five iteration for achieving good quality.

Table(1) Comparison of the images restored by the proposed algorithm with different iteration in term of PSNR (in decibel) value

\begin{tabular}{|c|c|c|c|c|c|c|c|c|}
\hline & & \multicolumn{6}{|c|}{ Noise density } & \multirow[b]{2}{*}{$70 \%$} \\
\hline Iteration & Images & $10 \%$ & $20 \%$ & $30 \%$ & $40 \%$ & $50 \%$ & $60 \%$ & \\
\hline \multirow[t]{3}{*}{1} & Lena & 50.90 & 50.32 & 49.07 & 46.74 & 43.61 & 39.13 & 35.33 \\
\hline & Baboon & 50.91 & 50.13 & 49.23 & 46.72 & 43.83 & 39.19 & 35.13 \\
\hline & cameraman & 50.90 & 50.04 & 49.07 & 46.99 & 43.66 & 39.67 & 35.76 \\
\hline \multirow[t]{3}{*}{2} & Lena & 50.87 & 50.25 & 49.05 & 47.79 & 46.50 & 43.67 & 39.15 \\
\hline & Baboon & 50.83 & 50.06 & 49.21 & 47.61 & 46.70 & 43.72 & 39.13 \\
\hline & cameraman & 50.80 & 49.92 & 49.05 & 47.85 & 46.36 & 39.67 & 35.76 \\
\hline \multirow[t]{3}{*}{3} & Lena & 50.76 & 49.73 & 48.87 & 47.83 & 46.74 & 45.42 & 41.70 \\
\hline & Baboon & 50.73 & 49.91 & 49.04 & 47.57 & 47.00 & 45.10 & 41.63 \\
\hline & cameraman & 50.68 & 49.69 & 48.77 & 47.86 & 46.76 & 45.50 & 42.06 \\
\hline \multirow[t]{3}{*}{4} & Lena & 50.65 & 49.31 & 48.69 & 47.78 & 46.75 & 45.75 & 43.31 \\
\hline & Baboon & 50.62 & 49.76 & 48.87 & 47.51 & 47.02 & 45.55 & 43.10 \\
\hline & cameraman & 50.56 & 49.51 & 48.56 & 47.80 & 46.78 & 45.86 & 43.37 \\
\hline \multirow[t]{3}{*}{5} & Lena & 50.54 & 49.15 & 48.50 & 47.73 & 46.71 & 45.77 & 44.13 \\
\hline & Baboon & 50.52 & 49.60 & 48.67 & 47.43 & 46.98 & 45.64 & 43.84 \\
\hline & cameraman & 50.42 & 49.30 & 48.34 & 47.78 & 46.75 & 45.97 & 44.07 \\
\hline
\end{tabular}




\subsection{Comparative Performance}

The comparative performance of all three filters with increasing amount of noise is given in the Table (2). According to the table proposed technique provides good quality of restored images as compared to the Fuzzy Logic Based Adaptive Noise Filter and CM filter. The given comparison of performance is given in term of PSNR values.

Table (2)

\begin{tabular}{|c|c|c|c|c|c|c|c|c|}
\hline \multirow[b]{2}{*}{ Methods } & \multirow[b]{2}{*}{ Images } & \multicolumn{7}{|c|}{ Noise Density } \\
\hline & & $10 \%$ & $20 \%$ & $30 \%$ & $40 \%$ & $\mathbf{5 0 \%}$ & $60 \%$ & $70 \%$ \\
\hline \multirow[t]{3}{*}{ Proposed } & Leena & 50.90 & 50.25 & 49.07 & 46.74 & 43.61 & 39.13 & 35.33 \\
\hline & Baboon & 50.91 & 50.13 & 49.23 & 46.72 & 43.83 & 39.19 & 35.13 \\
\hline & Cameraman & 50.90 & 50.04 & 49.04 & 46.99 & 43.66 & 39.67 & 35.76 \\
\hline \multirow[t]{3}{*}{ FLBANF } & Leena & 34.19 & 34.18 & 34.16 & 33.09 & 33.89 & 33.61 & 32.48 \\
\hline & Baboon & 30.67 & 30.65 & 30.62 & 30.59 & 30.54 & 30.31 & 29.88 \\
\hline & Cameraman & 35.31 & 35.28 & 35.17 & 34.95 & 34.51 & 34.53 & 33.22 \\
\hline \multirow[t]{3}{*}{$\mathbf{C M}$} & Leena & 35.40 & 34.46 & 33.33 & 31.65 & 29.27 & 27.47 & 25.77 \\
\hline & Baboon & 26.94 & 26.74 & 25.03 & 23.88 & 21.16 & 18.47 & 16.86 \\
\hline & Cameraman & 28.40 & 27.81 & 26.63 & 24.51 & 21.97 & 19.14 & 16.66 \\
\hline
\end{tabular}

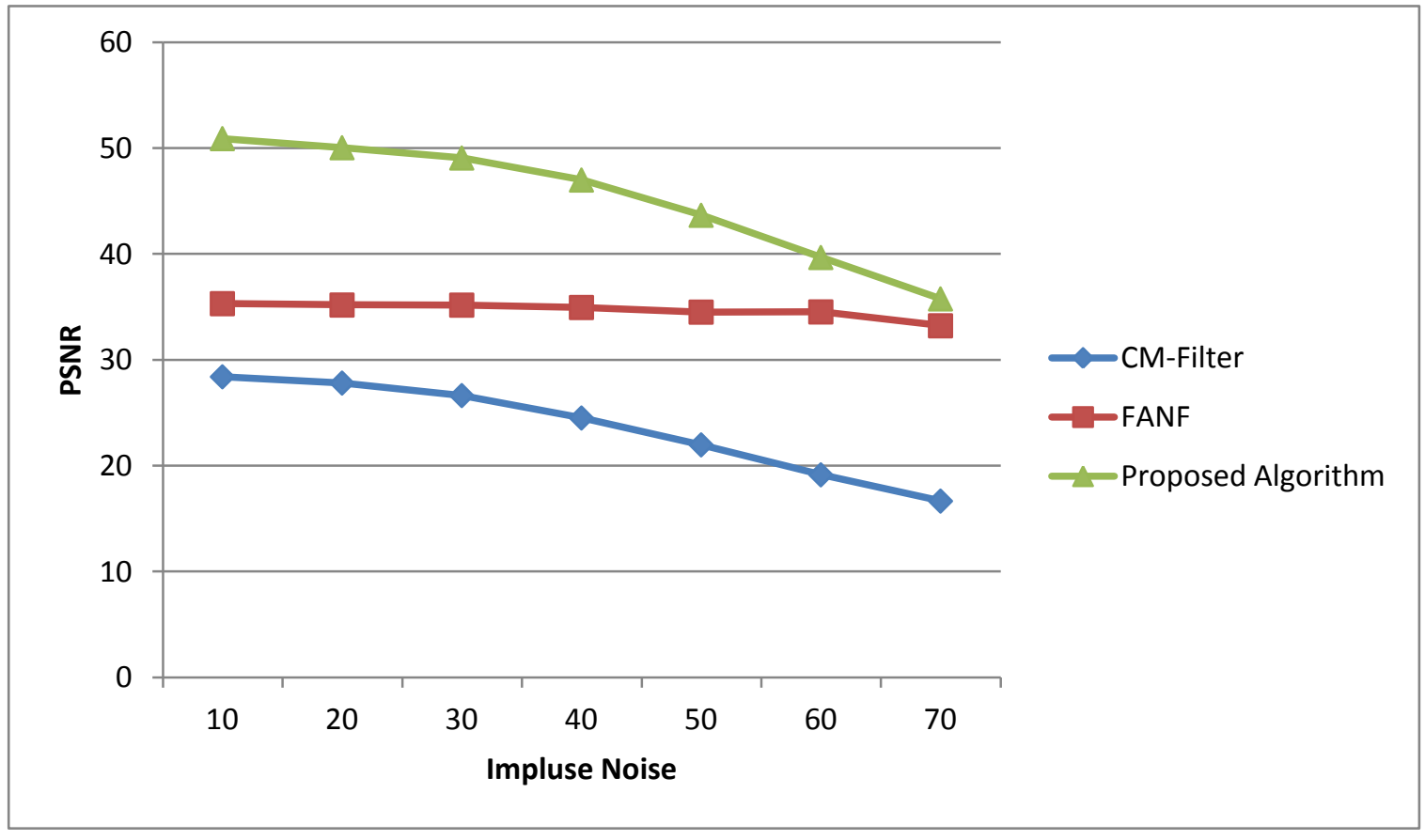

Fig (7) Comparison graph of PSNR at different noise density for Cameraman image 
The comparative performance of all three filters with increasing amount of noise is given in fig (7). According to the figure proposed technique provides good quality of restored images as compared to the Fuzzy Logic Based Adaptive Noise Filter and CM filter. The given comparison of performance is given in term of PSNR values. In this given diagram the performance of the CM filter is demonstrated using blue line which shows the least PSNR value as compared to other two filters. Additionally the performance of fuzzy logic based adoptive filter is given using red line which shows effective results as compared to the cloud model based impulse noise filter and shows less effectiveness as compared to the proposed filtering technique. The proposed impulse noise filtering technique's PSNR values are provided using green line which shows the higher PSNR values as compared to both traditionally found filters. In this diagram for simulating performance the different noise levels in image is given using $\mathrm{X}$ axis and their respective PSNR values are given in $\mathrm{Y}$ axis of graph.

\section{CONCLUSION}

In this presented study the impulse noise and their effect on digital images are investigated. In the number of image filtering algorithms, two promising algorithms are found in Jasdeep Kaur et al [1] and Zhe Zhou [2]. These algorithms are efficient and effective for removal of Impulse noise. But some of deficiencies are found in these techniques, therefore a new de-noising technique is proposed in this work. The proposed filter design includes the regression analysis for differentiating the corrupted and uncorrupted image pixels. Than after using uncorrupted neighbor pixels the corrupted pixels are replaced. This process is performed in iterative manner for removing the noise. On the other hand the processing of corrupted pixels can damage the edges of image thus a L0 gradient minimization technique is employed for preserving and smoothing the edges of image. Finally for removing the remaining noises and assuring the removal of noise from image the median filter is applied at the end of filtering process. This algorithm also provides good quality in higher percentage of noise, but algorithm require more iteration. In minimum no of iteration $1 \%$ or $2 \%$ noisy candidates are remain on image. In near future, the presented work is extended for developing more efficient technique that consumes less amount of time and less number of iterations during noise removal.

\section{ACKNOWLEDGEMENTS}

The authors would like to thank the reviewers for their helpful suggestions and contribution to improve the quality of the paper.

\section{REFERENCES}

[1] Jasdeep Kaur, Preetinder Kaur, Fuzzy Logic based Adaptive Noise Filter for Real Time Image Processing Applications, International Journal of Engineering Research \& Technology (IJERT), Vol. 1 Issue 7, September - 2012, ISSN: 2278-0181

[2] Zhe Zhou, Cognition and Removal of Impulse Noise With Uncertainty, IEEE TRANSACTIONS ON IMAGE PROCESSING, VOL.21, NO.7, JULY 2012.

[3] Jasdeep Kaur, Pawandeep Kaur, Preetinder Kaur, Review of impulse noise reduction technique using fuzzy logic for image processing, International Journal of Engineering Research \& Technology (IJERT), Vol. 1 Issue 5, July - 2012, ISSN: 2278-0181

[4] Ali S. Awad, Standard Deviation for Obtaining the Optimal Direction in the Removal of Impulse Noise, IEEE SIGNAL PROCESSING LETTERS, VOL. 18, NO. 7, JULY 2011

[5] S.Esakkirajan,T.veerakumar, Adabala N. Subramanyam, and C.H PremChand, Removal of High Density Salt and Pepper Noise Through Modified Decision Based Unsymmetric Trimmed Median Filter, IEEE SIGNAL PROCESSING LETTERS,VOL. 18,NO.5,MAY 2011

[6] S.Muthukumar, G.Raju, A Non-Linear Image Denoising Method for Salt-\&-pepper Noise Removal using FuzzyBased Approach, International Conference On Image Information Processing, 2011

[7] Kenny Kal Vin Toh, and Nor Ashidi Mat Isa, Noise Adaptive Fuzzy Switching Median Filter for Salt-andPepper Noise Reduction, IEEE SIGNAL PROCESSING LETTERS, VOL. 17, NO. 3, MARCH 2010

[8] Li Xu*, Cewu Lu*, Yi Xu ,Jiaya Jia, Image Smoothing via $L 0$ Gradient Minimization, Vol. 30, No. 6, December 2011.

[9] Di Guo, Xiaobo Qu, Xiaofeng Du, Keshou Wu, and Xuhui Chen, Hindawi Publishing Corporation Advance in Multimedia Volume 2014,Article ID 682747 Publishing Corporation.

[10] Ramanaiah N, Satish Kumar V, "Removal of High Density Salt and Pepper Noise in Images and Videos Using De-noising Methods", IJCSMC, Vol. 2, Issue. 10, October 2013, pg.234 - 242

[11] Xin Geng, Xiaoguang Hu, Jin Xiao, "Quaternion switching filter for impulse noise reduction in color image”, \& 2011 Elsevier B.V. All rights reserved. 